\title{
Diabetes Pathophysiology
}

\section{The Role of Advanced Glycation End-products in the Etiology of Insulin Resistance and Diabetes}

\author{
Helen Vlassara, MD ${ }^{1}$ and Gary E Striker, MD $^{2}$ \\ 1. Professor of Diabetes and Aging, and Professor of Medicine, Molecular Medicine, and Geriatrics, and Director, Division of Experimental Diabetes and Aging; \\ 2. Professor of Medicine, Department of Medicine and Department of Geriatrics, Mount Sinai School of Medicine
}

DOI: $10.17925 /$ USE.2010.06.1.14

\begin{abstract}
Despite new and effective drug therapies, insulin resistance (IR) and type 2 diabetes and its complications remain major medical challenges. It is known that IR, often associated with overnutrition and obesity, results from elevated oxidant stress (OS) and chronic inflammation. Less widely known is that a major cause for this inflammation is excessive consumption of advanced glycation end-products (AGEs) by the citizens of developed countries. AGES, which were largely thought as oxidative derivatives resulting from diabetic hyperglycemia, are increasingly seen as a potential risk factor for islet $\beta$-cell injury, peripheral IR, and diabetes. This article will discuss the relationships between exogenous AGEs, chronic inflammation, $\mathbb{R}$, and type 2 diabetes. We present new insights on the failure of innate immune defense mechanisms under chronic oxidant overload, which increase susceptibility to IR and type 2 diabetes and its complications. Finally, we describe evidence on AGE restriction, a new non-pharmacologic intervention, which effectively reduces persistent IR, restores innate immune functions, and, thus, optimizes current antidiabetic drug therapies.
\end{abstract}

\section{Keywords}

Glycation, oxidation, diet, inflammation, innate immunity, advanced glycation end-products (AGEs), AGE-receptors, advanced glycated end-product receptor 1 (AGER1), sirtuin (silent mating type information regulation 2 homolog) 1 (SIRT1)

Disclosure: The authors have no conflicts of interest to declare.

Received: August 18, 2010 Accepted: October 25, 2010 Citation: US Endocrinology, 2010;6:14-9

Correspondence: Helen Vlassara, MD, Division of Experimental Diabetes and Aging, Mount Sinai School of Medicine, Box 1640, One Gustave Levy Place, New York,

New York 10029. E: helen.vlassara@mssm.edu

The incidence of type 2 diabetes continues to increase at epidemic proportions, ${ }^{1}$ while vascular and renal diabetic complications have huge socioeconomic consequences. The etiology of this disease is multifactorial and an increasing amount of evidence points to environmental and lifestyle factors rather than genetic abnormalities as the most important predisposing factors. ${ }^{2,3}$ Type 2 diabetes is often accompanied by obesity and is characterized by inflammation and insulin resistance (IR), both of which are attributed to increased oxidant stress (OS).

While earlier clinical trials ${ }^{4,5}$ reinforced the importance of control of hyperglycemia in the pathogenesis of diabetic complications, more recent studies ${ }^{6-8}$ re-introduced the question of non-glucose-related risk factors in these pathologies. One of the pathways by which hyperglycemia causes cell injury is via the formation of advanced glycation end-products (AGES). In fact, glucose-derived AGEs still account for many of the complications of diabetes, largely by increasing systemic and intracellular OS. ${ }^{9-11}$

However, compelling data indicate that AGEs may cause beta cell injury ${ }^{12-15}$ and peripheral IR. ${ }^{16,17}$ This evidence led to the emergence of a potentially important view, that an excess of oxidants, such as AGEs in diets of the populations of developed countries, could play a previously unforeseen role in the initiation and progression of pre-diabetes to diabetes. ${ }^{18}$

\section{Endogenous Advanced Glycation End-products-Not Only from High Glucose}

The spontaneous chemical transformation of amine-containing molecules by reducing sugars is a process known as the Maillard reaction. Reducing sugars react non-enzymatically with free amino groups of proteins, lipids, and guanyl nucleotides in DNA to form adducts, which rearrange to form AGEs.

Among naturally occurring sugars, glucose exhibits the slowest glycation rate, while fructose, glucose-6-phosphate, glyceraldehyde-3-phosphate and threose form AGEs at a much faster rate. ${ }^{19}$ AGEs may also form with the amino groups of lipids ${ }^{20}$ and nucleic acids ${ }^{9}$ and the entire process is accelerated in diabetes and in conditions of chronic OS, as unopposed reactive oxygen species (ROS) are a potent trigger for glycoxidants.

Despite the identification of numerous AGE compounds that exist in nature, it is still unclear which are the most pathogenic AGEs. Pentosidine, carboxymethyllysine (CML), and methylglyoxal (MG)-derivatives are among the better-characterized compounds and these are often used as AGE markers. ${ }^{18,21,22}$ 


\section{Impact of Advanced Glycation End-products on Insulin Secreting and Insulin Sensitive Tissues}

A large body of literature points to the multiorgan injury caused by AGEs, implicating them in most complications associated with diabetes. ${ }^{2,9-11}$ However, other studies have began to suggest that AGES are involved in islet $\beta$-cell damage: aminoguanidine (AG), which inhibits the formation of $\mathrm{AGES}^{23}$ protected islet $\beta$-cell function in vivo ${ }^{24}$ as well as in isolated rat islets. ${ }^{24,25}$ Restriction of orally absorbed AGES significantly protected pancreatic islet morphology and/or function in four models of diabetes: mice susceptible to autoimmune type 1 diabetes (in NOD mice) or to type 2 diabetes $(d b / d b+/+ \text { mice })^{13,14}$ (see Figure 1), as well as fat-induced or age-related diabetes. ${ }^{15,17}$ Recent evidence affirmed these findings and assigned AGE-mediated $\beta$-cell toxicity to the inhibition of cytochrome-c oxidase and adenosine triphosphate (ATP) production, causing impaired insulin secretion. ${ }^{12,24,25}$ These studies began to fill in some of the conceptual gaps on the potential sources and mechanisms by which pre-existing oxidant overload can lead to $\beta$-cell insufficiency and damage.

Other findings shed more light at the other end of glucose-insulin interactions, supporting AGES as the cause of reduced peripheral insulin responsiveness and glucose utilization. Studies in adipocytes or mice, exposed to specific AGEs, showed that glycoxidants can lead to a decrease in insulin receptor and insulin-receptor substrate 1 (IRS-1) phosphorylation levels, consequently impairing glucose-uptake, via Jun $\mathrm{N}$-terminal kinase (JNK) activation. ${ }^{26}$ These findings have began to shift previously held views and to introduce the notion that prolonged exposure to oxidant overload-possibly spanning several generations - can deplete innate immune defense and foster defective insulin action, a possibility that has begun to receive support in the clinical setting. ${ }^{18,21,26}$

\section{Exogenous Sources of Advanced Glycation End-products-A New Cause of Diabetes?}

AGES are readily introduced into the circulation of the human body with nutrients ${ }^{27}$ or tobacco smoking ${ }^{28}$ long before, or in the absence of diabetes. Certain methods of food processing, particularly heat and dehydration, significantly accelerate the generation of new highly reactive dicarbonyl derivatives of complex glyco- and lipoxidation reactions. ${ }^{29,30}$ Approximately $10 \%$ of AGEs in a single AGE-rich meal are absorbed into the circulation, and two-thirds are still in the body seven hours later, ${ }^{31}$ an interval sufficient to promote tissue injury. AGES such as CML, MG-derivatives, and others are potent inducers of inflammation. ${ }^{30,32,33}$ As the effects of either exogenous or endogenous AGEs can be blocked by both antioxidants and anti-AGE agents, ${ }^{23,30,32}$ they in fact have a full range of overlapping biological properties (see Figure 2). The daily intake of AGEs with regular meals (e.g. of non-excessive caloric content) by healthy adults is estimated to exceed the 'safe' range by at least two to three times, ${ }^{34}$ related largely to the fact that the preferred methods of food preparation by the average US adult promote the AGE overload. This may also account for the alarming recent finding that high serum AGE levels in mothers correlated with those of their infants and predicted higher plasma insulin levels or homeostatic model assessment (HOMA) and lower adiponectin, ${ }^{35}$ factors that may pre-condition infants to high OS and, possibly, to diabetes.
Figure 1: Oral Advanced Glycation End-products and Islet Morphology

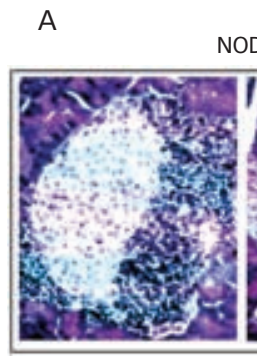

Regular diet
NOD

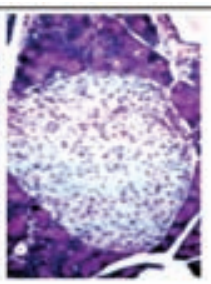

Low-AGE diet

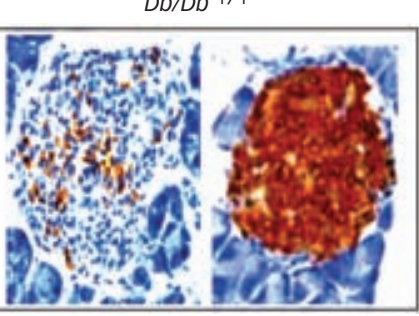

Regular diet

LOW-AGE diet
A: Non-obese diabetic (NOD) mice, ${ }^{13} \mathrm{~B}$ : Db/Db ${ }^{+/+}$mice ${ }^{14}$ were exposed to either regular or low advanced glycation end-products (AGE) diet (right panels). After a low-AGE diet (for $>12$ months) NOD mouse pancreatic tissues showed a striking absence of infiltrating mononuclear cells (right), typical in NOD fed a standard diet (generally AGE-rich, left panel) (magnification x400, hematoxylin and eosin stain [H\&E]). B: Islets of age-matched $\mathrm{Db} / \mathrm{Db}^{+/+}$mice, after a low-AGE diet (for five months; right), showed intact insulin production compared with those on regular diet (left) (magnification $x 400$, stained for insulin).

\section{Figure 2: Properties of Dietary Advanced} Glycation End-products
A

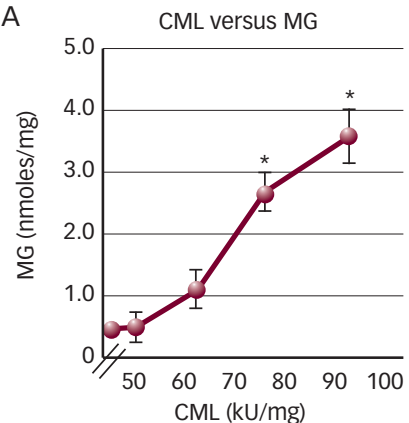

C

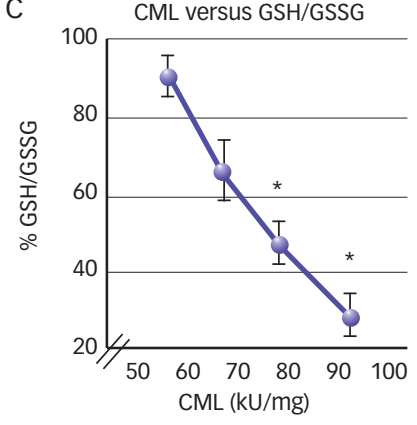

B

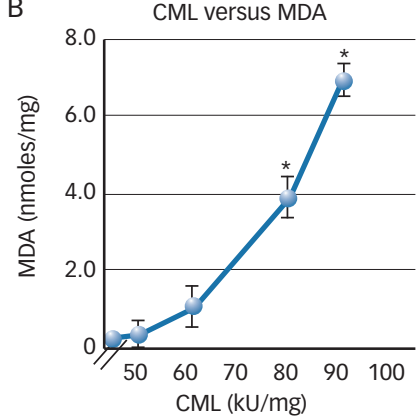

D Cytokine induction (TNF- $\alpha$ )

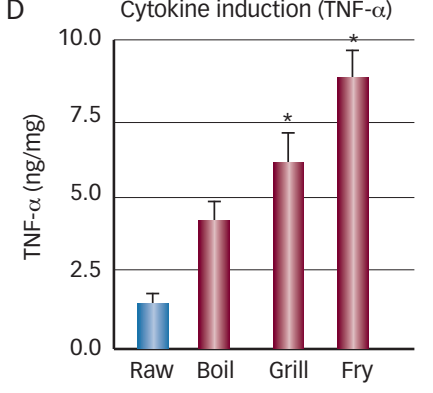

A: Correlation between food-derived protein advanced glycation end-products (AGE) (carboxymethyllysine [CML] and methylglyoxal [MG]-derivatives); B: Between CML and malondialdehyde (MDA) — a lipid oxidation product; C: Food-derived CML added to cultured endothelial cells $(E C)$ depletes intracellular levels of glutathione, consistent with increased oxidant stress; D: Levels of tumor necrosis factor-alpha (TNF- $\alpha$ ) in EC, after the addition of identical amounts of food extracts but differing in preparation method. ${ }^{37}$

$G S H=$ reduced g/utathione; $G S S G=$ oxidized g/utathione.

Two large databases containing the AGE content of common foods have been made available. ${ }^{34,36}$ These illustrate how dry heat-processing methods (such as broiling, searing, or frying) significantly increase the protein- and lipid-AGE content of foods compared with methods that utilize lower temperatures and moisture (i.e. stewing, steaming, or boiling; see Table 1) and how the same amount or type of nutrients, i.e. of fats can deliver dramatically different amounts of oxidant substances, 
Table 1: Thermally Modulated Advanced Glycation End-product Content in Common Food Items

\begin{tabular}{llllll} 
& \multicolumn{2}{l}{$\begin{array}{l}\text { Low Advanced Glycation } \\
\text { End-product Diet (U/mg) }\end{array}$} & & \multicolumn{2}{l}{ Regular diet (U/mg) } \\
Beef & Stewed & 2,000 & $\rightarrow$ & Broiled & 5,367 \\
\hline Chicken & Stewed & 1,011 & $\rightarrow$ & Broiled & 5,245 \\
\hline Salmon & Raw & 502 & $\rightarrow$ & Broiled & 1,358 \\
\hline Potato & Steamed & 17 & $\rightarrow$ & Fried & 1,522
\end{tabular}

Advanced glycation end-product (AGE) levels in foods, especially animal products, depend largely on the method used for its preparation. Data are shown as AGE carboxymethyllysine (CML) units per milligram of food, based on a enzyme-linked immunosorbent assay. ${ }^{34,35}$ Note the differences between modest and higher thermal exposure for identical amounts of nutrients/calories.

Figure 3: Properties of Advanced Glycated End-product Receptor 1

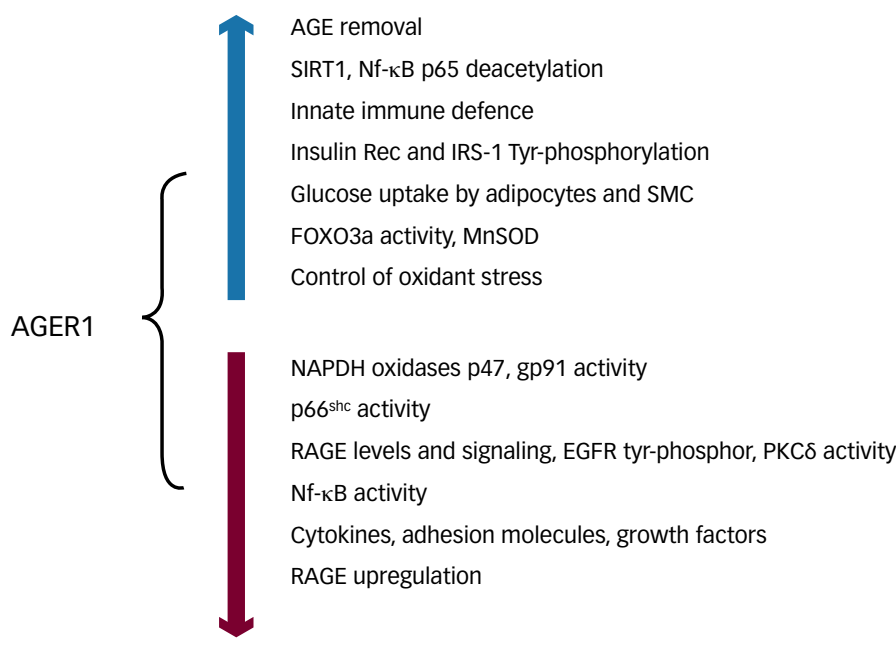

Advanced glycated end-product receptor 1 (AGER1) is an anti-AGE receptor with antioxidant stress (OS) functions. Insulin rec = insulin receptor; IRS-1 = insulin-receptor substrate 1; MnSod = manganese superoxide dismutase; $N A P D H=$ nicotinamide adenine dinucleotide: $N F-K B=$ nuclear factor kappa B: SIRT1 = sirtuin (silent mating type information regulation 2 homolog) 1; $P K C \delta=$ protein kinase $C \delta ; R A G E=$ receptor for $A G E ; S M C=$ smooth-muscle cell.

which may also promote antigenic stimulation. These databases helped in the development of tools for assessing dietary AGE intake, as well as for adjusting diets to a lower AGE content, while maintaining optimal caloric and nutrient intake. Given to subjects with or without diabetes or with chronic kidney disease, a low AGE dietary regimen proved highly effective in lowering circulating AGEs, inflammation, and IR. ${ }^{21,26}$ This intervention, if confirmed, may represent a significant advance toward the prevention of type 2 diabetes, or the improved efficacy of diabetes therapies. ${ }^{21,37}$

\section{Humans and Food Advanced Glycation End-products}

Significantly lower levels of serum AGEs were observed in patients (with type 1 and type 2 diabetes, after six weeks on a low-AGE American Diabetes Association (ADA)-recommended diet. ${ }^{37}$ This and several other studies demonstrated that the diet is a significant source of AGEs for humans and that orally absorbed AGEs directly affect serum AGE levels, and therefore, oxidant-antioxidant balance in patients with type 2 diabetes. Prior to these studies, elevated AGE levels in patients with type 2 diabetes had been attributed to endogenous sources, especially hyperglycemia and OS. ${ }^{9}$ In pursuing the origin of elevated OS further, it was noted that people without diabetes who consumed a diet with a high AGE content had levels of serum AGEs associated with diabetes and higher OS compared with those consuming a diet with a lower AGE content. ${ }^{18,21}$ Contrary to previous belief, serum AGEs were found to be independent of age, but correlated with established markers of OS and inflammation. ${ }^{18,21}$ In addition, there was a significant association between serum AGES and HOMA, an indicator of IR, in healthy subjects. Importantly, consumed AGES (but not calories, nutrients, glucose, or lipids) proved to be independent predictors of serum AGES and high-sensitivity $\mathrm{C}$-reactive protein (hSCRP), a prototypic marker of inflammation. These data suggested that the common diet is a significant source of oxidants in people without diabetes or in a pre-diabetic state. This may be of particular concern today since many 'healthy' subjects with a normal body mass index (BMI) tend to consume diets with excessive glycoxidants and may thus be at risk for type 2 diabetes.

\section{Advanced Glycation End-product Metabolism}

Steady-state serum AGE levels reflect the balance of oral intake, endogenous formation, and catabolism of AGES. AGE catabolism is dependent on both tissue degradation and renal elimination. At a tissue level, two main types of cellular AGE receptors are characterized. One of them, known as the receptor for AGEs (RAGE) although it recognizes many ligands otherthan AGEs, promotes and perpetuates cell activation and causes tissue injury via increased OS. ${ }^{38}$ An opposite type of receptor binds, degrades AGEs, and protects tissues from oxidant injury. The best evaluated in this protective category of AGE-receptors is advanced glycated end-product receptor 1 (AGER1), which has considerable antioxidant and anti-inflammatory properties based on studies on cells and mice overexpressing this gene $e^{21,39-43}$ (see Figure 3). The balance between these two receptors may be critical in the maintenance of oxidant homeostasis or progression to diabetes. Recent evidence suggests that AGER1 also acts to protect sirtuin (silent mating type information regulation 2 homolog) 1 (SIRT1), a major deacetylase and regulator of inflammation and insulin actions. SIRT1, similar to AGER1, is suppressed in chronic diabetes, but restored after lowering external oxidant burden by AGE restriction. ${ }^{21}$

AGE-peptides normally filter across the glomerular membrane and undergo variable degree of reabsorption and further catabolism by the renal proximal tubule, while the rest is excreted in the urine. ${ }^{44}$ While AGEs are also taken up by the proximal tubules from the blood and the interstitium, a precise and quantitative analysis of the contribution of each of these processes is lacking in humans. The important role of the kidneys in the metabolism and excretion of AGEs is demonstrated by an inverse correlation between serum AGE levels and renal function (glomerular filtration rate) and by the abnormal handling of an oral AGE load by animals and humans with severe renal disease. .7,45,46 $^{2}$

\section{The Importance of Anti-advanced Glycation End-product Receptors}

In healthy subjects, ambient levels of AGEs and ROS drive the expression of both AGER1 and RAGE. A short-term increase in AGEs is associated with an increase in RAGE, as well as in AGER1, which maintains the oxidative balance within cells. Similarly, consumption of a low-AGE diet by healthy subjects effectively lowers both AGER1 and RAGE levels. ${ }^{21}$ 
Importantly, AGER1 levels correlate inversely with the intracellular levels of AGES, and directly with urine AGEs in people without diabetes, consistent with its active role in AGE turnover and elimination by the normal kidney. Similar relationships have not been reported for RAGE nor soluble RAGE (SRAGE), although RAGE correlates with risk for cardiovascular disease (CVD) in patients with type 1 diabetes. ${ }^{21}$

The situation is quite different under conditions of chronically elevated AGEs, such as in type 2 diabetes or chronic kidney disease. Despite full antidiabetic therapy, RAGE levels remain high, while AGER1 levels are uniformly suppressed, 2,17,21,26,33 which are both indicative of persistent high OS. Interestingly, following four months on a low AGE diet, AGER1 levels were restored in patients with diabetes, while RAGE levels were significantly suppressed. ${ }^{21}$ This pattern suggests that depletion or loss of function of the AGER1 gene contributes to diabetic tissue injury, possibly by intracellular AGE accumulation, ROS generation, suppression of nicotinamide adenine dinucleotide (NAD+)-dependent SIRT1 and further increased ROS. Hyperactivation of RAGE, as well as of other pro-inflammatory gene products, is likely a consequence of the initial failure of defensive mechanisms, such as of AGER1 and SIRT1, to fend off oxidant overload, feeding into a cycle of OS and innate defense depletion.

While further studies are necessary, from these studies, one message is that under these conditions decisive headway may be gained only by restraining the currently unregulated entry of exogenous oxidants. Another message is that innate immune defenses and antioxidants may be rapidly bolstered after reducing the external oxidant load, rather than by continuing to increase the dosages of perfectly functional but inadequate oral antioxidants.

\section{Clinical Studies in Diabetes and Advanced Glycation End-product Toxicity}

There is considerable support for the role of AGEs in human diabetes, as well as for non-diabetic CVD or renal disease. ${ }^{47-51}$ A significant correlation was previously found between circulating AGE-apolipoprotein B (apoB) levels, vascular tissue AGEs, and severity of atherosclerotic lesions in patients without diabetes with coronary artery occlusive disease, ${ }^{48}$ and in patients with diabetes with aortic stiffness..$^{50}$ Serum pentosidine has also been shown to correlate positively with heart-brachial pulse wave velocity and with carotid intima-media thickness ${ }^{52}$ in patients with type 2 diabetes. In a random sample of Finnish patients with type 2 diabetes followed for 18 years, serum levels of AGEs were associated with total and CVD mortality in women. ${ }^{22}$ These studies reinforce the role of AGES in diabetic complications, apart from that of hyperglycemia.

Moreover, the concept that nutrient-derived oxidant AGEs could pose risk for acquiring diabetes_not only for diabetic vascular disease-has introduced a paradigm shift, according to which hyperglycemia could be the downstream effect, not the cause of high AGEs and thus, glucose control is but one variable that must be controlled (see Figure 4).

Without altering blood glucose or glycated hemoglobulin $\left(\mathrm{HbA}_{1 \mathrm{c}}\right)$ levels, a six-week AGE-restricted diet given to a group of patients with type I and $\|$ diabetes led to the significant reduction of indicators of inflammation and endothelial dysfunction, including hsCRP, tumor

\section{Figure 4: Serum Advanced Glycation End-product} Kinetics in Normal Adults
A. Normal subjects

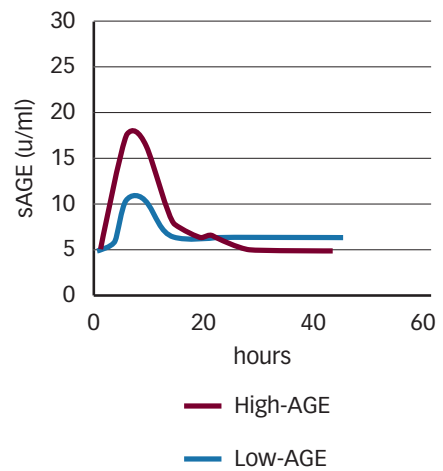

B. Diabetic subjects

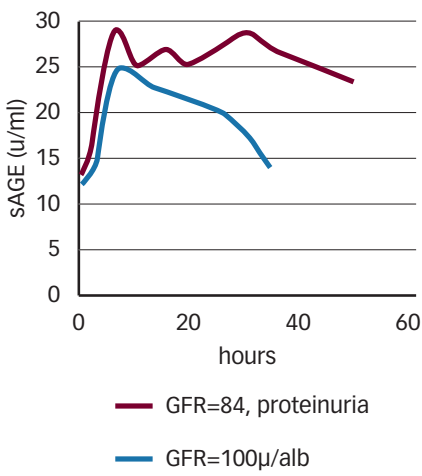

A: In healthy subjects, after a regular meal (high-advanced glycation end-products [AGE]) or a meal with lower AGES (low-AGE); B: Diabetic subjects with kidney disease given the same amount of AGES and calories. Note: a higher baseline and a more prolonged elevation of serum AGE (SAGE) levels (blue line) corresponds to those with significantly lower glomerular filtration rate (GFR), and thus with reduced urine AGE excretion (not shown). ${ }^{22}$ While neither group had severe renal disease AGE excretion was markedly decreased in both groups.

\section{Figure 5: People with Type 2 Diabetes}
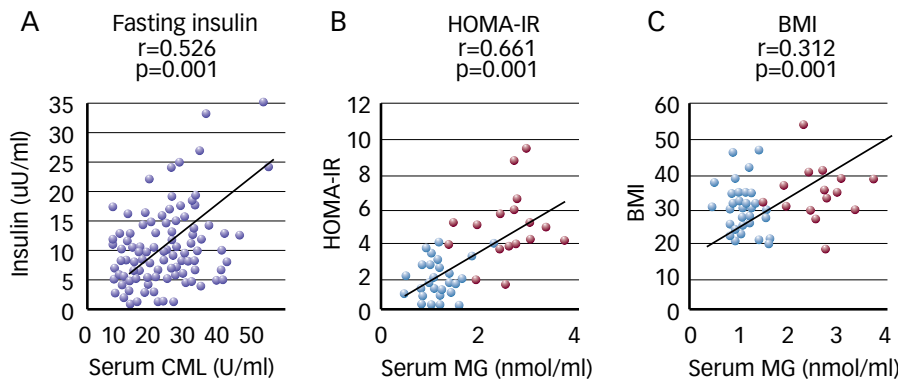

- Healthy Diabetes

Serum advanced glycation end-products (AGE) correlate with fasting plasma insulin (A), homeostatic model assessment insulin resistance (HOMA-IR) (B), and body mass index (BMI) (C). Serum AGES carboxymethyllysine (CML) or methylglyoxal (MG)-derivatives were assessed by enzyme-linked immunosorbent assays (ELISAS). Statistical significance is indicated. ${ }^{67}$

necrosis factor-alpha (TNF- $\alpha$ ), vascular cell adhesion molecule 1 (VCAM-1), and AGE levels. ${ }^{36,37}$ More recently, serum AGEs were shown to correlate with fasting insulin, homeostatic model assessment (HOMA)-IR, and $\mathrm{BMI}$ in patients with type 2 diabetes (see Figure 5). ${ }^{26}$ After a longer treatment interval of such patients with an AGE-restricted diet (four months), there was a significant reduction in plasma insulin, as well as in leptin, along with a marked rise in adiponectin. These changes were consistent with an improvement in insulin sensitivity in these patients with diabetes, who had persistent IR despite medical treatment. There were also a significant increase in AGER1 and SIRT1 levels and reduced nuclear factor-kappa B (NFKB) activity, based on decreased mononuclear TNF- $\alpha$, all consistent with suppressed inflammation in these subjects. Of note, this was the first study to show a dramatic reduction in RAGE levels ( 50-60\%) below baseline.

These findings await confirmation in larger trials. Since they were independent of other changes in medical therapy, they strongly support the postulate that it is the externally derived pro-inflammatory AGES that drive inflammation and IR to a large extent. ${ }^{21,26}$ They also show that 
these effects can be easily-and economically-modulated by a modest decrease $(\sim 50 \%)$ of the amount of AGEs in the diet and no changes in nutrients or calories.

An AGE challenge test diet produced a short-term endothelial dysfunction in both healthy subjects and patients with diabetes. ${ }^{53}$ It should be noted that the test beverage contained neither carbohydrates nor lipids, both of which are known to produce post-prandrial endotheilial dysfunction. Finally, a single AGE-rich solid meal induced a more pronounced acute impairment of vascular function than did an otherwise identical low AGE meal in patients with diabetes. ${ }^{54}$ These acute studies suggest that repeated or chronic exposure to inflammation/ROS from food-derived AGES could cause long-term vascular dysfunction, apart from excess glucose or lipids.

\section{Strategies to Reduce Dietary Advanced Glycation End-products}

After consuming a meal with high AGE content, healthy adults show a rapid absorption of AGES, with a rapidly rising peak in serum AGE level. ${ }^{27}$ A close correlation was observed between AGEs consumed and AGEs in the circulation in a cross-section of healthy subjects. ${ }^{18}$ In addition, studies in healthy patients with or without diabetes with chronic renal insufficiency showed that lowering dietary AGE intake (by 50\%) decreases circulating AGE levels as well as circulating markers of inflammation and OS. ${ }^{21,37,55}$ More importantly, the marked improvement in hyper-insulinemia in patients with type 2 diabetes on a low AGE diet, otherwise isocaloric, confirmed that exogenous AGES actively participate in the metabolic disturbances seen in type 2 diabetes. On a practical level, low dietary AGE intake can be easily achieved by adjusting the common methods of meal preparation to methods that use lower heat and higher humidity (e.g. instead of roasting, grilling, or frying to use stewing or poaching, etc.) and avoid pre-packaged and fast foods. ${ }^{34,36}$ Patients with diabetes have found that such a program is easily incorporated into their personal and family life.

\section{Countering Endogenous Advanced Glycation End-products, Not Only of Hyperglycemia}

Hyperglycemia is a major source of native AGES. Therefore, intensive treatment of hyperglycemia helps maintain control of AGEs. For instance, in a large cohort of type 1 diabetic patients from the Diabetes Control and Complications Trial (DCCT) skin collagen glycation, glycoxidation, and crosslinking were decreased in those undergoing long-term intensive treatment more than in patients on conventional treatment. ${ }^{54,56}$ While the focus on hyperglycemia was nearly exclusive in earlier clinical trials, ${ }^{4,57}$ recently conducted large studies (Action to Control Cardiovascular Risk in Diabetes [ACCORD], Action in Diabetes and Vascular Disease [ADVANCE], neoadjuvant androgen deprivation therapy [NADT] $)^{6,858}$ failed to produce anticipated conclusions. In addition, these did not deal with the transition from pre-diabetes to diabetes, highlighting the old question of non-traditional factors on precipitating diabetes and subsequent vascular complications. In view of the evidence discussed above, having not adjusted for exogenous oxidants or AGEs may have influenced the results. Well-controlled cellular and animal studies suggest that AGEs can incite beta cell injury ${ }^{12}$ and that the occurrence and severity of type 1 and type 2 diabetes diabetes and its complications depends on exogenous oxidant (AGE) overload rather than hyperglycemia. ${ }^{58}$

\section{Agents and Antioxidants that Prevent New Advanced Glycation End-product Formation}

A number of agents act by inhibiting post-Amadori advanced glycation reactions or by trapping carbonyl intermediates (glyoxal, MG, and 3-deoxyglucosone) and include aminoguanidine-hydrochloride form (HCL), ${ }^{59-61}$ benfotiamine ${ }^{62-64}$ and pyridoxamine. ${ }^{65,66}$ Other agents, such as ORB-9195, are thought to inhibit both glycation and lipoxidation reactions. ${ }^{67,64} \mathrm{~A}$ number of these are expected to become available in the near future.

Several studies have proposed various antioxidants as anti-AGE agents, including vitamin $\mathrm{E}^{, 69} \mathrm{~N}$-acetylcysteine, ${ }^{710}$ taurine, ${ }^{71}$ alpha lipoic acid, ${ }^{72}$ penicillamine, ${ }^{73}$ nicanartine, ${ }^{74}$ etc. More studies are needed to establish the effectiveness of antioxidants, even if the results from trials on a wide range of oral antioxidants have been disappointing, as in the past these were not informed by the current evidence and, thus, were not designed to address the large exogenous overload of oxidants.

\section{Conclusions}

There is little controversy surrounding the fact that AGEs contribute significantly to OS and inflammation in diabetes and complications associated with diabetes. Endogenous hyperglycemia is a significant driving force for the generation of AGES, but this can be substantially greater, since it is built on a premise of already elevated or pre-existing overt OS. It is becoming increasingly clear that the common diet is a carrier of AGEs and, thus, is a major contributor to basal OS, which impairs innate immune defenses and drives IR. This causal factor is of particular concern in subjects not only with diabetes but also with pre-diabetes. Thus, AGE overload as a cause of diabetes is an area of major clinical relevance. While many pharmacologic anti-AGE therapies are under development, their efficacy remains to be proved. In the interim, interventions that reduce both exogenous (food-derived) as well as endogenous (hyperglycemia-derived) AGES can provide definitive and lasting beneficial effects for diabetes and its complications.

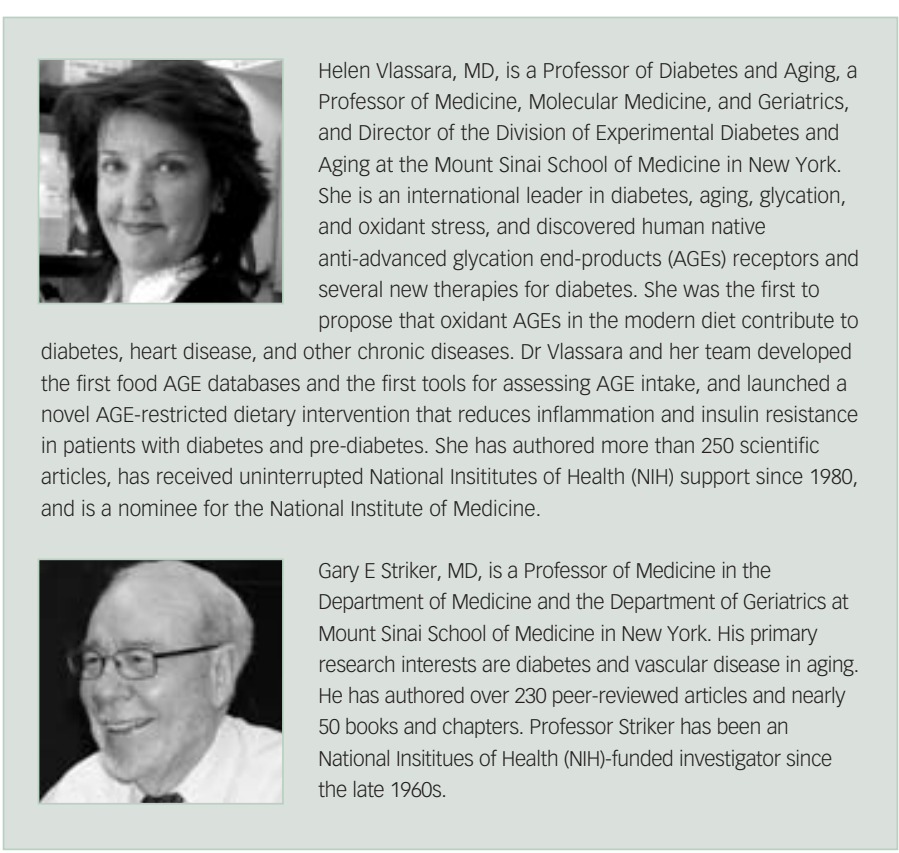


1. Amos AF, McCarty DJ, Zimmet P, Diabet Med, 1997;14 (Suppl. 5):S1-85.

2. Huebschmann AG, Regensteiner JG, Vlassara H, Reusch JE, Diabetes Care, 2006;29(6):1420-32.

3. Ford ES, Giles WH, Mokdad AH, Diabetes Care, 2004; 27(10): 2444-9.

4. The effect of intensive treatment of diabetes on the development and progression of long-term complications in insulin-dependent diabetes mellitus. The Diabetes Control and Complications Trial Research Group, N Engl J Med, 1993;329:977-86.

5. UK Prospective Diabetes Study (UKPDS) Group, Lancet, 1998; 352(9131):837-53.

6. Skyler JS, Bergenstal R, Bonow RO, et al., J Am Coll Cardiol 2009;53(3):298-304.

7. ACCORD Study Group, Ginsberg HN, Elam MB, et al., N Engl J Med, 2010;362(17):1563-74.

8. Duckworth W, Abraira C, Moritz T, et al., N Engl J Med, 2009;360(2):129-39.

9. Brownlee $\mathrm{M}$, Nature, 2001;414(6865):813-20.

10. Vlassara H, Palace MR, J Intern Med, 2002;251(2):87-101.

11. Thorpe SR Baynes JW, Drugs Aging, 1996; 9(2):69-77.

12. Zhao Z, Zhao C, Zhang XH, et al., Endocrinology, 2009;150(6): 2569-76.

13. Peppa M, He C, Hattori M, et al., Diabetes, 2003;52(6):1441-8

14. Hofmann SM, Dong HJ, Li Z, et al., Diabetes, 2002;51(7): 2082-9.

15. Sandu O, Song K, Cai W, et al., Diabetes, 2005;54(8):2314-9.

16. Cai W, He JC, Zhu L, et al., Am J Pathol, 2008;173(2):327-36.

17. Cai W, He JC, Zhu L, et al., Am J Pathol, 2007;170(6): 1893-1902.

18. Uribarri J, Cai W, Peppa M, et al., J Gerontol A Biol Sci Med Sci, 2007;62(4):427-33.

19. Suárez G, Rajaram R, Oronsky AL, Gawinowicz MA, J Biol Chem, 1989;264(7):3674-9.

20. Bucala R, Mitchell R, Arnold K, et al., J Biol Chem, 1995;270(18): 10828-32.

21. Vlassara H, Cai W, Goodman S, et al., I Clin Endocrinol Metab, 2009:94(11):4483-91.

22. Kilhovd BK, Juutilainen $A$, Lehto $S$, et al., Diabetologia, 2007:50(7):1409-17.

23. Brownlee $\mathrm{M}$, Vlassara $\mathrm{H}$, Kooney $\mathrm{A}$, et al., science, 1986:232(4758):1629-32

24. Tajiri Y, Moller C, Grill V, Endocrinology, 1997;138(1):273-80.

25. Tajiri Y, Grill V, Int J Exp Diabetes Res, 2000;1(2):111-9.

26. Jaime Uribarri WC, Ramdas M, Goodman S, et al., Improved Inflammation and Insulin Resistance by Restricting AGE
Intake; Potential Role of AGER1 and SIRT1, 2010; in press.

27. Koschinsky T, He CJ, Mitsuhashi T, et al., Proc Natl Acad Sci U S A, 1997;94(12):6474-9.

28. Cerami C, Founds H, Nicholl I, et al., Proc Natl Acad Sci U S A, 1997:94(25):13915-20.

29. O'Brien J, Crit Rev Food Sci Nutr, 1989;28:211-48.

30. Cai W, Gao QD, Zhu L, et al., Mol Med, 2002;8(7): 337-46.

31. He C, Sabol J, Mitsuhashi T, Vlassara H, Diabetes, 1999;48(6):1308-15.

32. Vlassara H, Fuh H, Makita Z, et al., Proc Natl Acad Sci U S A, 1992:89(24):12043-7.

33. Cai W, He JC, Zhu L, Chen X, et al., Am J Physiol Cell Physiol, 2008;294(1):C145-52

34. Uribarri J, Woodruff S, Goodman S, et al., J Am Diet Assoc, 2110;(6):911-6

35. Mericq V, Piccardo C, Cai W, et al., Diabetes Care, 2010;33(1): 2232-7.

36. Goldberg T, Cai W, Peppa M, et al., J Am Diet Assoc, 2004;104(8):1287-91.

37. Vlassara H, Cai W, Crandall J, et al., Proc Natl Acad Sci U S A, 2002;99(24):15596-601.

38. Yan SF, D'Agati V, Schmidt AM, Ramasamy R, Curr Mol Med, 2007;7(8):699-710

39. Cai W, He JC, Zhu L, et al., Proc Natl Acad Sci U S A, 2006 103(37):13801-6.

40. Cai W, Torreggiani M, Zhu L, et al., Am J Physiol Cell Physiol, 2010;298(3):C624-34

41. Lu C, He JC, Cai W, et al., Proc Natl Acad Sci U S A, 2004:101(32):11767-72

42. Torreggiani M, Liu H, Wu J, et al., Am J Pathol, 2009;175(4): $1722-32$

43. Yang Z, Makita Z, Horii Y, et al., J Exp Med, 1991;174(3): $515-24$

44. Saito A, Nagai R, Tanuma A, et al., J Am Soc Nephrol, 2003; 14(5):1123-31.

45. Vlassara H, Torreggiani M, Post JB, et al., Kidney Int Suppl, 2009(114):S3-11.

46. Makita Z, Radoff S, Rayfield EJ, et al., N Eng/ J Med, 1991;325(12):836-42.

47. Schleicher ED, Wagner E, Nerlich AG, J Clin Invest, 1997;99(3):457-68.

48. Yamada K, Miyahara Y, Hamaguchi K, et al., Clin Nephrol, 1994;42(6):354-61.

49. Sakata N, Imanaga Y, Meng J, et al., Atherosclerosis, 1999;142(1):67-77.

50. Stitt AW, He C, Friedman S, et al., Mol Med, 1997;3(9):
$617-27$

51. Stitt AW, Li YM, Gardiner TA, et al., Am J Pathol, 1997;150(2): 523-31.

52. Yoshida N, Okumura K, Aso Y, Metabolism, 2005;54(3): $345-50$

53. Uribarri J, Stirban A, Sander D, et al., Diabetes Care, 2007:30(10):2579-82.

54. Negrean M, Stirban A, Stratmann B, et al., Am J Clin Nutr, 2007:85(5):1236-43

55. Uribarri J, Peppa M, Cai W, et al., J Am Soc Nephrol, 2003;14(3):728-31.

56. Odetti P, Traverso N, Cosso L, et al., Diabetologia, 1996:39(12): $144-47$.

57. Keavney BD, Dudley CR, Stratton IM, et al., Diabetologia, 1995;38(8):948-52

58. Vlassara H, Striker G, Curr Diab Rep, 2007;7(3): 235-41.

59. Soulis T, Cooper ME, Vranes D, et al., Kidney Int, 1996;50(2): $627-34$

60. Panagiotopoulos S, O'Brien RC, Bucala R, et al., Atherosclerosis, 1998;136(1):125-31.

61. Kelly DJ, Gilbert RE, Cox AJ, et al., J Am Soc Nephrol 2001;12(10):2098-2107.

62. Pomero F, Molinar Min A, La Selva M, et al., Acta Diabetol 2001;38(3):135-8

63. Stracke $H$, Hammes HP, Werkmann D, et al. Exp Clin Endocrinol Diabetes, 2001;109(6):330-6

64. Thornalley PJ, Curr Diabetes Rev, 2005;1(3):287-98.

65. Voziyan PA, Hudson BG, Ann N Y Acad Sci, 2005;1043: 807-16.

66. Williams ME, Bolton WK, Khalifah RG, et al., Am I Nephrol, 2007;27(6):605-14

67. Williams ME, Bolton WK, Khalifah RG, et al., Diabetes, 1997:46(5):895-9

68. Wada R, Nishizawa Y, Yagihashi N, et al., Eur J Clin Invest, 2001;31(6):513-20

69. Odetti P, Robaudo C, Valentini S, et al., Contrib Nephrol, 1999:127:192-9.

70. Nakayama M, Izumi G, Nemoto Y, et al., Perit Dial Int, 1999:19(3):207-10.

71. Trachtman H, Futterweit S, Prenner J, Hanon S, et al., Biochem Biophys Res Commun, 1994:199(1):346-52.

72. Kunt T, Forst T, Wilhelm A, et al., Clin Sci (Lond), 1999;96(1):75-82

73. Jakus V, Hrnciarová M, Cársky J, et al., Life Sci, 1999;65(18-19): 1991-3.

74. Hammes HP, Bartmann A, Engel L, Wülfroth P, Diabetologia, 1997;40(6):629-34 